TITLE:

\title{
Biorthogonal approach for explicitly correlated calculations using the transcorrelated Hamiltonian
}

\author{
$\operatorname{AUTHOR}(S):$
}

Hino, O; Tanimura, Y; Ten-no, S

\section{CITATION:}

Hino, O ... [et al]. Biorthogonal approach for explicitly correlated calculations using the transcorrelated Hamiltonian. JOURNAL OF CHEMICAL PHYSICS 2001, 115(17): 7865-7871

\section{ISSUE DATE:}

2001-11-01

URL:

http://hdl.handle.net/2433/49967

\section{RIGHT:}

Copyright 2001 American Institute of Physics. This article may be downloaded for personal use only. Any other use requires prior permission of the author and the American Institute of Physics. 


\title{
Biorthogonal approach for explicitly correlated calculations using the transcorrelated Hamiltonian
}

\author{
Osamu Hino and Yoshitaka Tanimura \\ The Graduate University for Advanced Sciences and The Institute for Molecular Science, Myodaiji, \\ Okazaki 444-8585, Japan \\ Seiichiro Ten-no \\ Graduate School of Human Informatics, Nagoya University, Chikusa-ku Nagoya 464-8601, Japan
}

(Received 10 April 2001; accepted 14 August 2001)

\begin{abstract}
A biorthogonal formulation is applied to the non-Hermite transcorrelated Hamiltonian, which treats a large amount of the dynamic correlation effects implicitly. We introduce biorthogonal canonical orbitals diagonalizing the non-Hermitian Fock operator. We also formulate many-body perturbation theory for the transcorrelated Hamiltonian. The biorthogonal self-consistent field followed by the second order perturbation theory are applied to some pilot calculations including small atoms and molecules. (C) 2001 American Institute of Physics. [DOI: 10.1063/1.1408299]
\end{abstract}

\section{INTRODUCTION}

The importance of the electron correlation effects has been widely appreciated in $a b$ initio quantum chemistry. Despite the successful developments of highly reliable correlated methods like coupled-cluster theory, ${ }^{1}$ most numerical results are obtained with a finite number of one-electronic basis functions. The slow convergence of such traditional correlated methods is a direct consequence of the inability to describe the correlation cusp. ${ }^{2-5}$ The inclusion of explicitly correlated functions turned out to ameliorate this feature dramatically. ${ }^{6-10}$ The uses of explicitly correlated Gaussiantype geminals ${ }^{9-16}$ and the functions with explicitly linear $r_{12}$ behavior ${ }^{17-19}$ have been plugged into many-electron theories to provide highly accurate approaches. After the improvements of scaling and feasibility, the explicitly correlated methods will play the most important role in contemporary quantum chemistry for reliable energetics with wide variety including the potential energy surfaces of large molecules.

Recently, Ten-no proposed the simple use of a similarity transformed Hamiltonian, which is parameterized with a fixed two-electronic function (geminal). ${ }^{20,21}$ Hereafter the Hamiltonian is referred to as the transcorrelated Hamiltonian after the former terminology of Boys and Handy. ${ }^{22}$ The geminal is determined such that the singular behavior of the Coulomb potential is compensated at short interelectronic distances. The accompanying correlation factor reproduces the correlation cusp in the many-electronic wave function appropriately. The non-Hermitian nature of the transcorrelated Hamiltonian, however, makes the construction of a self-consistent field nontrivial. The problem was resolved by employing a modified Møller-Plesset (MP) partitioning ${ }^{20}$ with the usual hermite component of the Hartree-Fock model Hamiltonian. Approximate ground state energies were obtained at the second order perturbation level based on the pseudo-orbital theory with the partitioning.

In this paper, we propose an attractive alternative to treat the transcorrelated Hamiltonian using biorthogonal formulation, which has been applied to many electron theories with nonorthogonal orbitals. For instance, Mayer, Surján, and coworkers proposed a method called the chemical Hamiltonian approach (CHA) (Refs. 23, 24) to eliminate the basis set superposition error (BSSE). Gouyet discussed the intermolecular interactions ${ }^{25,26}$ using a nonhermite unperturbed Hamiltonian. Biorthogonal perturbation formulas were developed by Gouyet ${ }^{25,26}$ and by Surján and Mayer. ${ }^{27}$ Fuchikami and Block treated the exchange interactions between monomers representing the interaction Hamiltonian with nonorthogonal orbitals. ${ }^{28}$ Cantu and co-workers proposed an application to the valence bond (VB) theory. ${ }^{29}$ They discussed a group theoretical treatment to construct the VB states with definite spin symmetry from biorthogonal orbitals and evaluate matrix elements. Norbeck and McWeeny performed preliminary VB calculations with biorthogonal orbitals. $^{30}$

We shortly review how operators are represented in the second quantized form using biorthogonal formulation in Sec. II. In Sec. III, we illustrate the transcorrelated Hamiltonian approach and discuss the self-consistent field theory using biorthogonal orbitals. In Sec. IV, we develop MBPT for the transcorrelated Hamiltonian based on the biorthogonal formalism. In Sec. V, we report numerical results. We also explain the approximate calculations of three-electron integrals, which appear in the transcorrelated method. We summarize this work in Sec. VI.

\section{BIORTHOGONAL SECOND QUANTIZATION}

The biorthogonal second quantization has been discussed in several literatures. ${ }^{23,26,28,29,31}$ We briefly show the key features of the formulation. Let us suppose the biorthogonal functions,

$$
\begin{aligned}
& \lambda=\left\{\chi_{1}, \chi_{2}, \ldots\right\}, \\
& \pi=\left\{\phi_{1}, \phi_{2}, \ldots\right\}, \\
& \int \chi_{p}(1) \phi_{q}(1) d 1=\delta_{p q},
\end{aligned}
$$


where the indices, $p, q, \ldots$, denote spin orbitals. For simplicity, the one-electronic functions are assumed to be real. The field operators are expressed in terms of the biorthogonal functions, which span the complete one-electronic space as,

$$
\begin{aligned}
& \Psi^{+}(1)=\sum_{p} b_{p}^{+} \chi_{p}(1), \\
& \Psi(1)=\sum_{q} c_{q} \phi_{q}(1),
\end{aligned}
$$

where $b_{p}^{+}$and $c_{q}$ are new creation and annihilation operators corresponding to the biorthogonal functions, $\phi_{p}$ and $\chi_{q}$, respectively,

$$
\begin{aligned}
& b_{p}^{+}=\int \phi_{p}(1) \Psi^{+}(1) d 1, \\
& c_{q}=\int \chi_{q}(1) \Psi(1) d 1 .
\end{aligned}
$$

These definitions of the operators are mathematically equivalent to the ones using the inverse of overlap integrals of the nonorthogonal basis set. ${ }^{23,26,28,29,31}$ One can express operators in the second quantized form using the biorthogonal basis functions. The one-electron operator,

$$
O^{1}=\sum_{i} \psi^{1}(i)
$$

is rewritten as

$$
\begin{aligned}
& O^{1}=\int \Psi^{+}(1) \omega^{1}(1) \Psi(1) d 1=\sum_{p q} \omega_{p q}^{1} b_{p}^{+} c_{q}, \\
& \omega_{p q}^{1}=\int \chi_{p}(1) \omega^{1}(1) \phi_{q}(1) d 1 .
\end{aligned}
$$

Similarly, the two-electron operator,

$$
O^{2}=\sum_{i>j} \omega^{2}(i, j)
$$

becomes

$$
\begin{aligned}
& O^{2}=\frac{1}{2} \sum_{p q r s} \omega_{p q r s}^{2} b_{p}^{+} b_{q}^{+} c_{s} c_{r}, \\
& \omega_{p q r s}^{2}=\iint \chi_{p}(1) \chi_{q}(2) \omega^{2}(1,2) \phi_{r}(1) \phi_{s}(2) d 1 d 2 .
\end{aligned}
$$

The Hermitian conjugate of $c_{p}$ is not $b_{p}^{+}$unless the orbital $\chi_{p}$ coincides with $\phi_{p}$. The usual anticommutation relations hold for the biorthogonal operators,

$$
\begin{aligned}
& \left\{b_{p}^{+}, b_{q}^{+}\right\}=0, \\
& \left\{c_{p}, c_{q}\right\}=0, \\
& \left\{b_{p}^{+}, c_{q}\right\}=\delta_{p q} .
\end{aligned}
$$

The Wick theorem can be used for the calculation of operator products. This fact enables us to use the conventional second quantized formalism, i.e., the normal ordering and diagrammatic techniques, for different determinatal states as different bracket vacuums. ${ }^{25,26,29}$ In what follows, we formulate a many-body perturbation theory for the transcorrelated method.

\section{TRANSCORRELATED HAMILTONIAN}

The transcorrelated Hamiltonian ${ }^{10,22,32}$ is defined as

$$
\widetilde{H}=e^{-F} H e^{F},
$$

which deals with the dynamic correlation effects accurately through the correlation factor, $e^{F}$. The correlation factor is dependent on the positions of electrons explicitly. The transformation does not change the energy spectrum while the effective Hamiltonian becomes non-Hermitian. This means that the variational calculation does not necessarily give the upper bound of the exact energy. The feature never becomes a major disadvantage in practical applications. In the recent development, ${ }^{20,21}$ a spherically symmetric geminal

$$
F=\sum_{i>j} f\left(r_{i j}\right)
$$

is used to describe the Coulomb hole in the vicinity of $r_{12}$ $=0$. Residual correlation effects are dealt with by the standard expansions with one-electronic functions. $\widetilde{H}$ is expanded in a power series of $F$ as

$$
\widetilde{H}=H+[H, F]+\frac{1}{2 !}[[H, F], F],
$$

which terminates at the double commutator. ${ }^{22,32}$ We can rewrite the transcorrelated Hamiltonian as

$$
\widetilde{H}=H+K+L,
$$

where the operators, $K$ and $L$, are in the biorthogonal form,

$$
\begin{aligned}
K & =\frac{1}{2} \sum_{p q r s}\left\langle p q\left|K_{12}\right| r s\right\rangle b_{p}^{+} b_{q}^{+} c_{s} c_{r}, \\
L & =\frac{1}{6} \sum_{p q r s t u}\left\langle p q r\left|L_{123}\right| s t u\right\rangle b_{p}^{+} b_{q}^{+} b_{r}^{+} c_{u} c_{t} c_{s},
\end{aligned}
$$

with the operators,

$$
\begin{aligned}
K_{12}= & -\nabla_{1}^{2} f\left(r_{12}\right)-\nabla_{1} f\left(r_{12}\right) \cdot \nabla_{1} f\left(r_{12}\right) \\
& -\nabla_{1} f\left(r_{12}\right) \cdot\left(\nabla_{1}-\nabla_{2}\right), \\
L_{123}= & -\nabla_{1} f\left(r_{12}\right) \cdot \nabla_{1} f\left(r_{13}\right)-\nabla_{2} f\left(r_{23}\right) \cdot \nabla_{2} f\left(r_{21}\right) \\
& -\nabla_{3} f\left(r_{31}\right) \cdot \nabla_{3} f\left(r_{32}\right) .
\end{aligned}
$$

We parameterize the geminal using the least square fitting such that the relation,

$$
\nabla_{1}^{2} f\left(r_{12}\right)+\nabla_{1} f\left(r_{12}\right) \cdot \nabla_{1} f\left(r_{12}\right) \cong r_{12}^{-1} w\left(r_{12}\right),
$$

holds approximately, ${ }^{20}$ where $w\left(r_{12}\right)$ is a short-range weight function. Under this condition, we localize the correlation factor to keep the additional integrals, Eqs. (3.7) and (3.8), increasing linearly to the system size, maintaining the proper description of the Coulomb cusp. The geminal is represented by a linear combination of Gaussian functions, 


$$
f\left(r_{12}\right)=-\sum_{G}^{N_{G}} c_{G} \exp \left(\zeta_{G} r_{12}^{2}\right) .
$$

We use another weight Gaussian function, $\exp \left(-\zeta_{w} r_{12}^{2}\right)$ for closed expressions in the least-square fitting. The present method does not treat the correlation factor as a variational parameter unlike the others ${ }^{6-15}$ including the original transcorrelated method. ${ }^{10,22}$ This feature bypasses the complicated nonlinear optimization of the factor, which requires four electron integrals. The antisymmetrized part of the wave function is treated efficiently with one-electronic basis functions since the transcorrelated Hamiltonian is almost free from the Coulomb singularity. The expansion series is expected to converge much faster than the usual wave function one.

We introduce the self-consistent field theory for the transcorrelated Hamiltonian using the biorthogonal formalism. Henceforward, the indices, $i, j, \ldots$, and $a, b, \ldots$, denote occupied and virtual orbitals in the biorthogonal basis, respectively. Let us suppose the conjugate states consisting of $N$ biorthogonal occupied orbitals,

$$
\begin{aligned}
& X=\left\|\chi_{1} \chi_{2} \cdots \chi_{N}\right\|, \\
& \Phi=\left\|\phi_{1} \phi_{2} \cdots \phi_{N}\right\|,
\end{aligned}
$$

with the normalization condition, $\langle X \mid \Phi\rangle=1$. The variation to the energy functional,

$$
\bar{E}_{\mathrm{SCF}}=\langle X|\widetilde{H}| \Phi\rangle,
$$

leads to the Brillouin theorem for the transcorrelated Hamiltonian,

$$
\begin{aligned}
& \left\langle X\left|\widetilde{H} b_{a}^{+} c_{i}\right| \Phi\right\rangle=\widetilde{f}_{i a}=0, \\
& \left\langle X\left|b_{i}^{+} c_{a} \widetilde{H}\right| \Phi\right\rangle=\widetilde{f}_{a i}=0 .
\end{aligned}
$$

The non-Hermite Fock operator is given by

$$
\begin{aligned}
\widetilde{f}_{p q}= & h_{p q}+\sum_{i}\left\langle p i\left|r_{12}^{-1}+K_{12}\right| q i\right\rangle_{A} \\
& +\frac{1}{2} \sum_{i j}\left\langle p i j\left|L_{123}\right| q i j\right\rangle_{A},
\end{aligned}
$$

where $h_{p q}$ are the one-electron integrals in the biorthogonal basis and the antisymmetrized matrix elements are

$$
\begin{aligned}
\left\langle p q\left|r_{12}^{-1}+K_{12}\right| r s\right\rangle_{A}= & \left\langle p q\left|r_{12}^{-1}+K_{12}\right| r s\right\rangle-\langle p q| r_{12}^{-1} \\
& +K_{12}|s r\rangle, \\
\left\langle p q r\left|L_{123}\right| s t u\right\rangle_{A}= & \left\langle p q r\left|L_{123}\right| s t u\right\rangle+\left\langle p q r\left|L_{123}\right| t u s\right\rangle \\
& +\left\langle p q r\left|L_{123}\right| u s t\right\rangle-\left\langle p q r\left|L_{123}\right| s u t\right\rangle \\
& -\left\langle p q r\left|L_{123}\right| t s u\right\rangle-\left\langle p q r\left|L_{123}\right| u t s\right\rangle .
\end{aligned}
$$

We can obtain the biorthogonal canonical orbitals by diagonalizing the Fock operator,

$$
\widetilde{f}_{p q}=\varepsilon_{p} \delta_{p q} .
$$

The choice of the model Hamiltonian fulfills the HellmanFeynmann theorem,

$$
\left.\frac{\partial}{\partial \alpha}\langle X(\alpha)|\widetilde{H}(\alpha)| \Phi(\alpha)\rangle\right|_{a=0}=\left\langle X\left|\frac{\partial \widetilde{H}}{\partial \alpha}\right| \Phi\right\rangle .
$$

Therefore, molecular properties like the energy gradients are simply calculated in terms of the transformed molecular integrals and the reduced density matrix for the biorthogonal states, $X$ and $\Phi$. One disadvantage is that we have to manipulate the time-consuming three-electron integrals iteratively. Fortunately, our choice of the short-ranged geminal makes the contribution of $L$ much less important than $K$ and the approximate form of the model Hamiltonian,

$$
\tilde{f}_{p q} \cong h_{p q}+\sum_{i}\left\langle p i\left|r_{12}^{-1}+K_{12}\right| q i\right\rangle_{A},
$$

works pretty well as will be discussed in Sec. V.

\section{MANY-BODY PERTURBATION FOR THE TRANSCORRELATED HAMILTONIAN}

Let us proceed with reviewing the biorthogonal perturbation theory. ${ }^{25-27,29}$

We derive perturbation formulas for the transcorrelated Hamiltonian following the previous biorthogonal theories. ${ }^{25-27,29}$ Suppose the partitioning,

$$
\widetilde{H}=\widetilde{H}_{0}+\widetilde{V} .
$$

The biorthogonal Slater determinants are assumed to be the eigenfunctions of the non-Hermitian model Hamiltonian, $\widetilde{H}_{0}$,

$$
\begin{aligned}
& \left(\widetilde{H}_{0}-E_{0}\right)|\Phi\rangle=0, \\
& \langle X|\left(\widetilde{H}_{0}-E_{0}\right)=0 .
\end{aligned}
$$

The projection of the Schrödinger equation,

$$
(\widetilde{H}-E) \Omega|\Phi\rangle=0,
$$

on the bra vector, $\langle X|$, gives the perturbation energy,

$$
E-E_{0}=\langle X|\widetilde{V} \Omega| \Phi\rangle,
$$

where $\Omega$ is the wave operator. Using the projection operators,

$$
\begin{aligned}
& P=|\Phi\rangle\langle X|, \\
& Q=1-P,
\end{aligned}
$$

and Eq. (4.4), we have

$$
\Omega=1+\frac{1}{z-\widetilde{H}_{0}} Q(z-E+\widetilde{V}) \Omega,
$$

for any number, $z$. Iterating the equation, we obtain the perturbative formulas for the wave operator and energy,

$$
\begin{aligned}
& \Omega=\sum_{m=0}^{\infty}\left[\frac{1}{z-\widetilde{H}_{0}} Q(z-E+\widetilde{V})\right]^{m}, \\
& E-E_{0}=\sum_{m=0}^{\infty}\left\langle X\left|\widetilde{V}\left[\frac{1}{z-\widetilde{H}_{0}} Q(z-E+\widetilde{V})\right]^{m}\right| \Phi\right\rangle .
\end{aligned}
$$


Setting the parameter, $z=E_{0}$, we obtain the biorthogonal Rayleigh-Schrödinger series, ${ }^{26,27}$

$$
E-E_{0}=\sum_{m=0}^{\infty}\left\langle X|\widetilde{V}|\left[\frac{1}{E_{0}-\tilde{H}_{0}} Q\left(E_{0}-E+\widetilde{V}\right)\right]^{m} \mid \Phi\right\rangle .
$$

Alternatively, we have the Brillouin-Wigner one with $z=E$,

$$
E-E_{0}=\sum_{m=0}^{\infty}\left\langle X\left|\widetilde{V}\left(\frac{1}{E-\tilde{H}_{0}} Q \widetilde{V}\right)^{m}\right| \Phi\right\rangle .
$$

Applying the partitioning,

$$
\begin{aligned}
\widetilde{H}_{0}= & \sum_{p q} \widetilde{f}_{p q} b_{p}^{+} c_{q}=\sum_{p} \varepsilon_{p} b_{p}^{+} c_{p}, \\
\widetilde{V}= & -\sum_{p q}\left(\widetilde{f}_{p q}-h_{p q}\right) b_{p}^{+} c_{q}+\frac{1}{2} \sum_{p q r s}\langle p q| r_{12}^{-1} \\
& +K_{12}|r s\rangle b_{p}^{+} b_{q}^{+} c_{s} c_{r} \\
& +\frac{1}{6} \sum_{p q r s}\left\langle p q r\left|L_{123}\right| s t u\right\rangle b_{p}^{+} b_{q}^{+} b_{r}^{+} c_{u} c_{t} c_{s},
\end{aligned}
$$

to the formula, Eq. (4.11), we obtain the second order perturbation energy,

$\bar{E}^{(2)}=\bar{E}_{D}^{(2)}+\bar{E}_{T}^{(2)}$,

$\bar{E}_{D}^{(2)}=\frac{1}{4} \sum_{a b i j} \frac{\widetilde{g}_{i j a b} \widetilde{g}_{a b i j}}{\varepsilon_{i}+\varepsilon_{j}-\varepsilon_{a}-\varepsilon_{b}}$,

$\bar{E}_{r}^{(2)}=\frac{1}{36} \sum_{a b c i j k} \frac{\left\langle i j k\left|L_{123}\right| a b c\right\rangle_{A}\left\langle a b c\left|L_{123}\right| i j k\right\rangle_{A}}{\varepsilon_{i}+\varepsilon_{j}+\varepsilon_{k}-\varepsilon_{a}-\varepsilon_{a}-\varepsilon_{b}}$,

where we defined the matrix elements

$\widetilde{g}_{p q r s}=\left\langle p q\left|r_{12}^{-1}+K_{12}\right| r s\right\rangle_{A}+\sum_{i}\left\langle p q i\left|L_{123}\right| r s i\right\rangle_{A}$.

If we use the approximate form of the Fock operator (3.21), the singles correction appears in the energy expression,

$$
\begin{aligned}
& \bar{E}^{(2)}=\bar{E}_{S}^{(2)}+\bar{E}_{D}^{(2)}+\bar{E}_{T}^{(2)}, \\
& \bar{E}_{S}^{(2)}=\frac{1}{4} \sum_{a i j k} \frac{\left\langle i j k\left|L_{123}\right| a j k\right\rangle_{A}\left\langle a j k\left|L_{123}\right| i j k\right\rangle_{A}}{\varepsilon_{i}-\varepsilon_{a}} .
\end{aligned}
$$

Usually, the triples energy, $\bar{E}_{T}^{(2)}$, is negligibly small for our localized geminal.

\section{NUMERICAL METHOD AND RESULTS}

For the application of the present biorthogonal method, the manipulation of the three-electron integrals is crucial because the explicit calculation of the integrals requires a large amount of CPU time and disk space. To avoid this difficulty, the approximate completeness insertion,

$$
\begin{aligned}
& \left\langle p q r\left|\nabla_{1} f_{12} \cdot \nabla_{1} f_{123}\right| s t u\right\rangle \\
& \cong \sum_{w}\left\langle p q\left|\nabla_{1} f_{12}\right| w t\right\rangle \cdot\left\langle w r\left|\nabla_{1} f_{12}\right| s u\right\rangle,
\end{aligned}
$$

was used in the previous work. ${ }^{20}$ The formula however requires all of the Cartesian components of the integrals, $\left\langle p q\left|\nabla_{1} f\left(r_{12}\right)\right| r s\right\rangle$. Furthermore, it is difficult to adapt the spatial symmetry. In this work, we employ an alternative formula. ${ }^{33}$ The operator, $K_{12}$, is decomposed into the linear and quadratic terms in the geminal as,

$$
\begin{aligned}
& K_{12}=\frac{1}{2}\left(K_{12}^{L}+K_{21}^{L}\right)+K_{12}^{Q}, \\
& K_{12}^{L}=-\nabla_{1}^{2} f_{12}-2 \nabla_{1} f_{12} \cdot \nabla_{1}, \\
& K_{12}^{Q}=-\nabla_{1} f_{12} \cdot \nabla_{1} f_{12} .
\end{aligned}
$$

Then the three-electron integrals are approximated by

$$
\begin{aligned}
\left\langle p q r\left|\nabla_{1} f_{12} \cdot \nabla_{1} f_{13}\right| s t u\right\rangle \cong & \frac{1}{2} \sum_{w}\left(\left\langle p r\left|K_{12}^{L}\right| w u\right\rangle\left\langle w q\left|f_{12}\right| s t\right\rangle\right. \\
& \left.-\left\langle p q\left|f_{12}\right| w t\right\rangle\left\langle w r\left|K_{12}^{L}\right| s u\right\rangle\right) .
\end{aligned}
$$

The operators, $K_{12}^{1}$ and $f_{12}$, are antisymmetric and symmetric to reduce the disk storage and to enable us to use the molecular symmetries. In addition to these features, the expression increases the accuracy of the completeness insertion. This is because the operators do not include extra momentum unlike the one, $\nabla_{1} f_{12}$, and do not increase the necessary angular momentum of the expansion functions, $w$. We consistently use the spherical harmonic basis functions for all the calculations.

\section{A. Beryllium atom}

We first apply the present biorthogonal $\operatorname{MBPT}(2)$ to the beryllium atom. The frozen geminal determined for $\zeta_{w}=5$ with 6-component Gaussian functions ${ }^{20}$ is employed throughout this work for all molecules consist of light atomic elements. We use the primitive functions in the atomic natural orbitals (ANO) triple zeta set, ${ }^{35} 14 s 9 p 4 d 3 f$, and check the convergence by removing the angular components outer than the $s$-shell. The approximate expression of the Fock operator (3.21) is used for the self-consistent scheme.

We compare the biorthogonal energies with the conventional MBPT (2) results in Table I. The component, $\Delta \bar{E}_{\mathrm{SCF}}$ $+E_{S}^{(2)}$, can be attributed to the single determinant contribution of the reference to the correlation energy, where $\Delta \bar{E}_{\mathrm{SCF}}=\left\langle X\left|\widetilde{H}-E_{\mathrm{HF}}\right| \Phi\right\rangle$. The singles energy, $E_{S}^{(2)}$, is less than $0.01 \mathrm{~m} E_{\mathrm{h}}$. This rationalizes the use of the approximate Fock operator. The triples contribution, $E_{T}^{(2)}$, is always less than $1 \mu E_{\mathrm{h}}$ and can be neglected practically. The reference

TABLE I. Biorthogonal MBPT energies of the beryllium atom $\left(\mathrm{m} E_{\mathrm{h}}\right)$.

\begin{tabular}{lcrccc}
\hline \hline \multicolumn{1}{c}{ Basis set } & $\Delta \bar{E}_{\mathrm{SCF}}+\bar{E}_{S}^{(2)}$ & \multicolumn{1}{c}{$\bar{E}_{D}^{(2)}$} & $\bar{E}_{T}^{(2)}$ & $\Delta \bar{E}_{\mathrm{SCF}}+\bar{E}^{(2)}$ & $\mathrm{MBPT}(2)$ \\
\hline $14 s$ & 58.38 & 6.46 & $0.1 \times 10^{-4}$ & 64.84 & 15.91 \\
$14 s 9 p$ & 58.38 & 18.82 & $1.2 \times 10^{-4}$ & 77.20 & 64.05 \\
$14 s 9 p 4 d$ & 58.38 & 22.34 & $1.9 \times 10^{-4}$ & 80.72 & 68.29 \\
$14 s 9 p 4 d 3 f$ & 58.38 & 23.33 & $1.9 \times 10^{-4}$ & 81.71 & 69.45 \\
\hline \hline
\end{tabular}

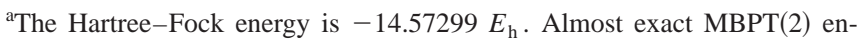
ergy is $-76.36 \mathrm{~m} E_{\mathrm{h}}$ (Ref. 39). The best R12-MBPT(2) and R12-CCSD(T) energies are -76.25 and $-94.29 \mathrm{~m} E_{\mathrm{h}}$, respectively (Ref. 19). 
TABLE II. Perturbation energies $\left(\mathrm{m} E_{\mathrm{h}}\right)$ of the methane molecule. ${ }^{\mathrm{a}}$

\begin{tabular}{ccccccc}
\hline \hline$\Delta \bar{E}_{\mathrm{SCF}}$ & $\bar{E}_{S}^{(2)}$ & $\bar{E}_{D}^{(2)}$ & $\Delta \bar{E}_{\mathrm{SCF}}+\bar{E}^{(2)}$ & $\mathrm{MBPT}(2)$ & $\mathrm{CCSD}$ & $\mathrm{CCSD}(\mathrm{T})$ \\
\hline 159.28 & 2.07 & 121.21 & 282.56 & 249.75 & 270.71 & 277.54 \\
$(59.03)$ & $(2.07)$ & $(-6.81)$ & & & & \\
\hline
\end{tabular}

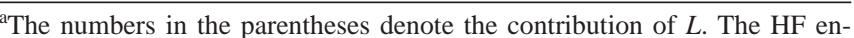
ergy is $-40.21469 E_{\mathrm{h}}$. The R12-MBPT(2)-A, R12-CCSD, and R12$\operatorname{CCSD}(\mathrm{T})$ correlation energies are $-273.58,-288.56$, and $-295.95 \mathrm{~m} E_{\mathrm{h}}$, respectively (Ref. 19).

energy, $\Delta \bar{E}_{\mathrm{SCF}}+E_{S}^{(2)}$, covers $\approx 70 \%$ of the total correlation energy and the residual is dealt with mainly by the doubles, $E_{D}^{(2)}$. The best biorthogonal MBPT(2) energy lies in between the exact MBPT(2) and R12-CCSD(T) ones. This is because the transcorrelated results implicitly include the quadruples and higher excitations, which are similar to the disconnected products in the coupled-cluster theory, as a result of the exponential form of the correlation factor. We expect a clear improvement by inclusion of higher order contributions ${ }^{33,34}$ because the nondynamic correlation effects are important for the atom.

\section{B. Methane and acetylene}

The methane molecule was calculated using the modified MP partitioning ${ }^{20}$ in the previous work. We apply the biorthogonal theory to the system to compare with the conventional results including those of the R12 theory. Additionally, we also calculate the acetylene molecule whose extrapolated $\operatorname{MBPT}(2), \operatorname{CCSD}$, and $\operatorname{CCSD}(\mathrm{T})$ energies are available. ${ }^{36}$ The primitive basis functions are derived from the parent basis set, cc-pVTZ of Dunning. ${ }^{37}$ To improve the description around core, we augment the basis set for carbon with $s-, p-$, and $d$-primitives with the exponents, 41615.0, 70.588 , and 3.784305 , respectively, which are even-tempered sequences of the original basis set. Other conditions follow the previous calculation.

In Table II, we show the result of methane. The calculations are performed with the geometrical parameters, $R_{\mathrm{CH}}$ $=1.0848 \AA$ and $\angle \mathrm{HCH}=109.47^{\circ}$. The conventional $\operatorname{MBPT}(2)$ energy is about $25 \mathrm{~m} E_{\mathrm{h}}$ above the R12$\operatorname{MBPT}(2)-\mathrm{A}$ one, whereas the present $\Delta \bar{E}_{\mathrm{SCF}}+\bar{E}^{(2)}$ is $-282.56 \mathrm{~m} E_{\mathrm{h}}$, which is in between R12-MBPT(2)-A and $\mathrm{R} 12-\mathrm{CCSD}(\mathrm{T})$. This agrees with the previous transcorrelated result using the modified MP partitioning. ${ }^{20}$ The singles energy, $-2.07 \mathrm{~m} E_{\mathrm{h}}$, is purely from the operator $L$ and is sufficiently small to be treated perturbationally outside the itera-
TABLE III. Perturbation energies $\left(\mathrm{m} E_{\mathrm{h}}\right)$ of the acetylene molecule. ${ }^{\mathrm{a}}$

\begin{tabular}{lcccccc}
\hline \hline$\Delta \bar{E}_{\mathrm{SCF}}$ & $\bar{E}_{S}^{(2)}$ & $\bar{E}_{D}^{(2)}$ & $\Delta \bar{E}_{\mathrm{SCF}}+\bar{E}^{(2)}$ & $\mathrm{MBPT}(2)$ & $\operatorname{CCSD}$ & $\operatorname{CCSD}(\mathrm{T})$ \\
\hline 296.28 & 2.95 & 173.02 & 472.24 & 411.58 & 425.50 & 443.40 \\
$(109.15)$ & $(2.95)$ & $(-14.37)$ & & & & \\
\hline \hline
\end{tabular}

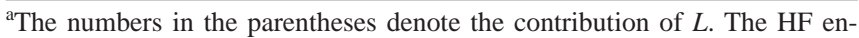
ergy is $-76.85194 E_{\mathrm{h}}$. The extrapolated correlation energies are -455.0 , -460.6 , and $-480.2 \mathrm{~m} E_{\mathrm{h}}$, at $\operatorname{MBPT}(2), \mathrm{CCSD}$, and $\operatorname{CCSD}(\mathrm{T})$, respectively (Ref. 36).

tive scheme. The contribution of $L$ in $\Delta \bar{E}_{\mathrm{SCF}}$, which is the first order correction with the approximate Fock operator, is somewhat larger in comparison with the beryllium case according to the increase of the number of electrons in the carbon atom.

The result of acetylene is given in Table III. The geometrical parameters used are $R_{\mathrm{CC}}=1.2033 \AA$ and $R_{\mathrm{CH}}$ $=1.0605 \AA$. In contrast to the methane result, the biorthogonal MBPT(2) energy is only $8 \mathrm{~m} E_{\mathrm{h}}$ above the R12-CCSD(T) one, since acetylene is strongly correlated in comparison with methane due to the presence of the triple bond. Evaluating the contrast improvement over the conventional methods, it is clear that the present transcorrelated Hamiltonian considerably enhances the accuracy with such a minor modification of the correlated method. In the subsequent publication, ${ }^{33,34}$ we will show the importance of higher-order energies in achieving the chemical accuracy.

\section{Water and neon}

The application of the transcorrelated Hamiltonian has been even straightforward for systems consisting of light atomic elements. As the mass of a constituent atom increases, however, we have to pay attention to the radius of the correlation factor. This is because the linear behavior of the correlation factor, ${ }^{4} f\left(r_{12}\right)=\frac{1}{2} r_{12}$, is adequate only in the slowly varying limit of the wave function. If the momentum difference of electrons is large, the term, $-\nabla_{1} f\left(r_{12}\right) \cdot\left(\nabla_{1}\right.$ $-\nabla_{2}$ ), in $K_{12}$ can become a primary perturbation over the Coulomb repulsion at somewhat large interelectronic distances. This makes the second-order treatment inappropriate especially for pair correlations including core electrons. Furthermore, the uncertainty in the completeness insertion increases according to the dominance of $L$ as described in the previous work. ${ }^{20}$ For instance, the three-body contribution to the total energy becomes as large as $-1000 \mathrm{~m} E_{\mathrm{h}}$ for Ne with the geminal derived in the previous study. In order to reduce the uncertainty from the completeness insertion for $L$, we

TABLE IV. Perturbation energies $\left(\mathrm{m} E_{\mathrm{h}}\right)$ of water. ${ }^{\mathrm{a}}$

\begin{tabular}{lcccccr}
\hline \hline \multicolumn{1}{c}{ Basis set } & $\mathrm{HF}$ & $\Delta \bar{E}_{\mathrm{SCF}}+\bar{E}_{S}^{(2)}$ & $\Delta \bar{E}_{\mathrm{SCF}}+\bar{E}^{(2)}$ & MBPT(2) & CCSD & CCSD (T) \\
\hline $9 s 4 p 1 d$ & 76030.43 & 103.16 & 317.27 & 257.74 & 266.34 & 271.24 \\
$10 s 5 p 3 d 1 f$ & 76057.32 & 101.40 & 347.04 & 318.46 & 325.06 & 333.40 \\
$12 s 6 p 5 d 3 f 1 g$ & 76064.89 & 101.12 & 354.88 & 341.90 & 345.74 & 355.27 \\
$14 s 8 p 7 d 5 f 3 g$ & 76066.87 & 101.05 & 356.23 & 348.89 & 351.48 & 361.36 \\
\hline \hline
\end{tabular}


$\operatorname{CCSD}(\mathrm{T})$ energies are $-361.691,-359.312$, and $-369.228 \mathrm{~m} E_{\mathrm{h}}$, respectively (Ref. 19). The Gaussian-type geminals MBPT(2) is $-356.43 \mathrm{~m} E_{\mathrm{h}}$ (Ref. 40). 


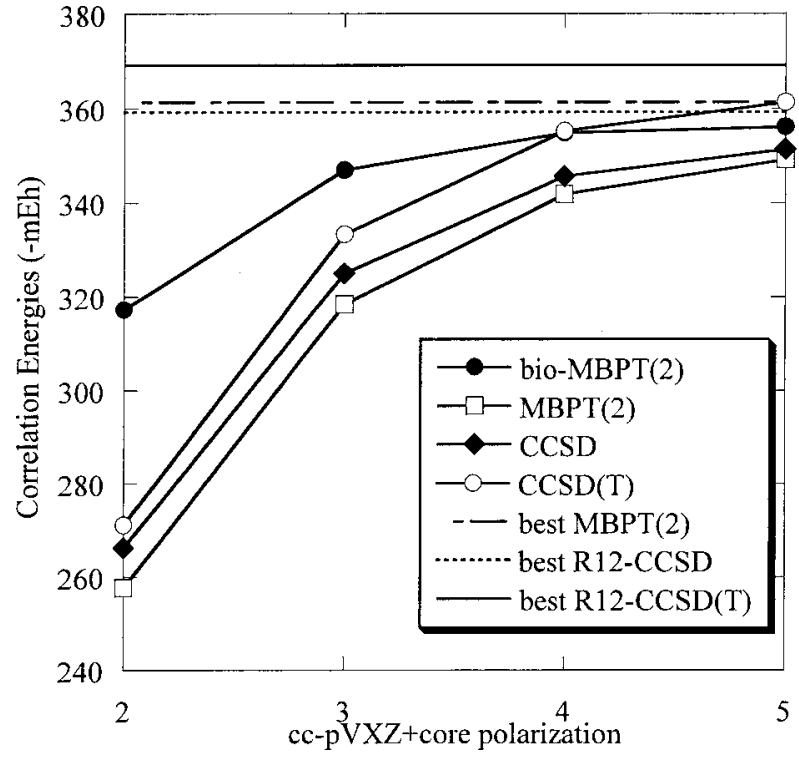

FIG. 1. Correlation energies of water for the cardinal number $\mathrm{X}$ in the cc-pVXZ basis set series augmented with the core polarization functions of cc-pCVXZ. Bio-MBPT(2), MBPT(2), CCSD, and CCSD(T) mean the biorthogonal MBPT(2), conventional MBPT(2), CCSD, and $\operatorname{CCSD}(\mathrm{T})$, respectively.

parameterize a tight geminal for systems including heavy elements using the weight exponent, $\zeta_{\mathrm{w}}=20$. The three-body contribution does not exceed $-140 \mathrm{~m} E_{\mathrm{h}}$ for the present systems under this condition. We use an even-tempered sequence of 6 Gaussian-type functions with the range of Gaussian exponents between 9040.0 and 0.52 . We also investigate the basis set convergence for water and neon using primitive functions for the heavy atoms derived from the parent basis sets, cc-pVXZ (Ref. 37) ( $X=D, T, Q$, and 5). The primitive sets are augmented with the $d-, f-$, and $g$-core polarization functions in the corresponding cc-pCXZ (Ref. 38) sets. This gives rise to the $9 s 4 p 1 d, 10 s 5 p 3 d 1 f$, $12 s 6 p 5 d 3 f 1 g$, and $14 s 8 p 7 d 5 f 3 g$ sets, respectively. The corresponding primitive sets from cc-pVXZ are used for hydrogen with the exception that cc-pVQZ is used for the largest oxygen set, $14 s 8 p 7 d 5 f 3 g$. The contributions of $\bar{E}_{T}^{(2)}$ are estimated for $10 s 5 p 3 d 1 f$. We do not discuss the effect in more detail since the absolute values of $\bar{E}_{T}^{(2)}$ are less than $0.01 \mathrm{~m} E_{\mathrm{h}}$ under the above conditions.

The correlation energies of the water molecule are shown in Table IV and Fig. 1. We use the bond length and bond angle, $R_{\mathrm{OH}}=0.9573 \AA$ and $\angle \mathrm{HOH}=104.52^{\circ}$. It is shown that the biorthogonal MBPT(2) energies yield consid-



FIG. 2. Correlation energies of neon for the cardinal number $X$ in the cc-pVXZ basis set series augmented with the core polarization functions of cc-pCVXZ. Bio-MBPT(2), MBPT(2), CCSD, and CCSD(T) mean the biorthogonal MBPT(2), conventional MBPT(2), CCSD, and $\operatorname{CCSD}(\mathrm{T})$, respectively.

erable improvements over the conventional MBPT(2). One notes the single determinant contribution, $\Delta \bar{E}_{\mathrm{SCF}}+\bar{E}_{S}^{(2)}$, is much less sensitive to the basis set in comparison with the change of the HF energy. The biorthogonal MBPT(2) energies is always superior to the conventional $\operatorname{MBPT}(2)$ and CCSD for the present basis sets. The $\operatorname{CCSD}(\mathrm{T})$ however gains more correlation energy at $12 s 6 p 5 d 3 f 1 g$ due to the importance of connected triples which are absent in the transcorrelated $\operatorname{MBPT}(2)$. The energy difference between the $10 s 5 p 3 d 1 f$ and $14 s 8 p 7 d 5 f 3 g$ sets is only $9 \mathrm{~m} E_{\mathrm{h}}$ in our approach, which is less than one-third of the conventional MBPT(2). This manifest improvements in the converge behavior is due to the absence of Coulomb singularity in the transcorrelated Hamiltonian.

We also show the results for the neon atom in Table $\mathrm{V}$ and Fig. 2. The correlation energies of the biorthogonal MBPT(2) are closer to the R12-CCSD(T) one than those of the conventional MBPT(2), CCSD, and CCSD(T). The best biorthogonal MBPT(2) energies of water and neon are very close to those of Gaussian-type geminals MBPT(2). The accuracy of the present fit for the correlation factor is $95.7 \%$, which is less than the previous one, $98.7 \%$, in which both the

TABLE V. Perturbation energies $\left(\mathrm{m} E_{\mathrm{h}}\right)$ of $\mathrm{Ne} .^{\mathrm{a}}$

\begin{tabular}{lcccccr}
\hline \hline \multicolumn{1}{c}{ Basis set } & HF & $\Delta \bar{E}_{\mathrm{SCF}}+\bar{E}_{S}^{(2)}$ & $\Delta \bar{E}_{\mathrm{SCF}}+\bar{E}^{(2)}$ & MBPT(2) & CCSD & CCSD(T) \\
\hline $9 s 4 p 1 d$ & 128488.78 & 190.99 & 366.31 & 255.48 & 257.48 & 259.64 \\
$10 s 5 p 3 d 1 f$ & 128531.86 & 187.69 & 381.17 & 330.53 & 332.81 & 337.68 \\
$12 s 6 p 5 d 3 f 1 g$ & 128543.47 & 183.66 & 385.31 & 360.63 & 361.84 & 368.85 \\
$14 s 8 p 7 d 5 f 3 g$ & 128546.77 & 183.47 & 385.60 & 370.69 & 370.78 & 377.24 \\
\hline \hline
\end{tabular}

${ }^{a}$ Almost exact MBPT(2) energy is $-387.9 \mathrm{~m} E_{\mathrm{h}}$ (Ref. 42). The R12-MBPT(2)-A, R12-CCSD, and R12$\operatorname{CCSD}(\mathrm{T})$ energies are $-388.311,-383.823$, and $-390.508 \mathrm{~m} E_{\mathrm{h}}$, respectively (Ref. 19). The Gaussian-type geminals MBPT(2) is $-385.3 \mathrm{~m} E_{\mathrm{h}}$ (Ref. 43). 
Gaussian exponents and coefficients are optimized. The quality of the fit may lead to a difference less than $10 \mathrm{~m} E_{\mathrm{h}}$ in the $\Delta \bar{E}_{\mathrm{SCF}}$ energy. Our preliminary calculations however showed that the total energy is much less sensitive to the accuracy of the fit. It is expected that the combinations with more sophisticated correlated methods like $\operatorname{CCSD}(\mathrm{T})$ will enable us to attain a calculation with a few $\mathrm{m} E_{\mathrm{h}}$ accuracy easily.

\section{CONCLUSIONS}

We have presented the biorthogonal formalism in our transcorrelated method. The best feature of the method is that the usual second quantization formalism over the biorthogonal basis sets can be used with the definition of canonical orbitals for the transcorrelated Hamiltonian. This makes the construction of single reference MBPT straightforward. The applications to the atoms and molecules showed that the present method leads to markedly better convergence improving the feasibility of the explicitly correlated calculations. All of the effective interactions in the transcorrelated Hamiltonian are parameterized to be short-ranged. This feature will make the application to even large molecules possible, with high accuracy in combination with the linearscaling methods. ${ }^{44-47}$

The contribution of the three-electron integrals becomes more important as the masses of the constituent atoms increase. In such a situation, we will have to evaluate the threeelectron integrals more precisely. The explicit evaluation of the three-electron integrals is feasible ${ }^{21}$ when the system size is sufficiently small. Our choice of the short-range geminal, however, implies that the integrals must be calculated explicitly only in the vicinity of the core electrons. The partial use of the explicit calculation along with the completeness insertion will be promising in the calculations with heavy atomic elements.

Finally, we mention the computational requirements of the present transcorrelated method. $N, V$, and $O$ are the numbers of basis functions, virtual orbitals, and occupied orbitals, respectively. The small contributions of the threeelectron integrals allow us to exclude the treatment of $L$ in the SCF cycle. The biorthogonal MBPT(2) calculation is dominated by generating the connected doubles which requires $\mathrm{NV}^{2} \mathrm{O}^{3}$ floating point operations. In this way, the original scaling of the correlated method is hardly altered by the introduction of the transcorrelated Hamiltonian.

We are implementing other correlated methods using the transcorrelated Hamiltonian, like MRCI and higher order perturbation schemes including the coupled cluster method. We will be able to report further investigations in these lines. $^{34}$

\section{ACKNOWLEDGMENTS}

The present work is partly supported by the Grant-inAids for Scientific Research on Priority Areas (A2) (No. 12042233) from the Ministry of Education, Culture, Sports, Science, and Technology, and the Grant-in-Aids for Scientific Research B (No. 13440177) from JSPS in Japan.
${ }^{1}$ R. J. Bartlett, "Coupled-cluster theory: An overview of recent developments," in Molecular Electronic Structure Theory, edited by D. R. Yarkony (World Scientific, Singapore, 1995), Vol. 2, and references therein.

${ }^{2}$ T. Kato, Commun. Pure Appl. Math. 10, 151 (1957).

${ }^{3}$ M. Hoffmann-Ostenhof and R. Seiler, Phys. Rev. A 23, 21 (1981).

${ }^{4}$ W. Kutzelnigg, Theor. Chim. Acta 68, 445 (1985).

${ }^{5}$ R. N. Hill, J. Chem. Phys. 83, 1173 (1985).

${ }^{6}$ E. A. Hyleraas, Z. Phys. 54, 347 (1929).

${ }^{7}$ H. M. James and A. S. Coolidge, J. Chem. Phys. 1, 825 (1933).

${ }^{8}$ W. Kolos and L. Wolniewicz, J. Chem. Phys. 41, 3663 (1964); 43, 2429 (1965).

${ }^{9}$ K.-C. Pan and H. F. King, J. Chem. Phys. 56, 4667 (1972).

${ }^{10}$ N. C. Handy, Mol. Phys. 26, 169 (1973).

${ }^{11}$ L. Adamowicz and A. J. Sadlej, Chem. Phys. Lett. 48, 305 (1977).

${ }^{12}$ L. Adamowicz and A. J. Sadlej, J. Chem. Phys. 67, 4298 (1977).

${ }^{13}$ K. Szalewicz, B. Jeziorski, H. J. Monkhorst, and J. G. Zabolitzky, J. Chem. Phys. 78, 1420 (1983); K. Szalewicz, J. G. Zabolitzky, B. Jezioski, and H. J. Monkhorst, ibid. 81, 2723 (1984).

${ }^{14}$ W. Cencek and J. Rychlewski, J. Chem. Phys. 98, 1252 (1993).

${ }^{15}$ R. Bukowski, B. Jeziorski, and K. Szalewicz, J. Chem. Phys. 110, 4165 (1999).

${ }^{16}$ B. J. Persson and P. R. Taylor, J. Chem. Phys. 105, 5915 (1996).

${ }^{17}$ W. Kutzelnigg and W. Klopper, J. Chem. Phys. 94, 1985 (1991); V. Termath, W. Klopper, and W. Kutzelnigg, ibid. 94, 2002 (1991); W. Klopper and W. Kutzelnigg, ibid. 94, 2020 (1991).

${ }^{18}$ J. Noga and W. Kutzelnigg, J. Chem. Phys. 101, 7738 (1994); J. Noga, D. Tunega, W. Klopper, and W. Kutzelnigg, ibid. 103, 309 (1995).

${ }^{19}$ J. Noga, W. Klopper, and W. Kutzelnigg, "CC-R12: An explicitly correlated coupled-cluster theory," in Recent Advances in Coupled-Cluster Methods, edited by R. J. Bartlett (World Scientific, Singapore, 1997).

${ }^{20}$ S. Ten-no, Chem. Phys. Lett. 330, 169 (2000).

${ }^{21}$ S. Ten-no, Chem. Phys. Lett. 330, 175 (2000).

${ }^{22}$ S. F. Boys and N. C. Handy, Proc. R. Soc. London, Ser. A 310, 43 (1969).

${ }^{23}$ I. Mayer, Int. J. Quantum Chem. 23, 341 (1983).

${ }^{24}$ P. R. Surján, I. Mayer, and I. Lukovits, Chem. Phys. Lett. 119, 538 (1985); P. R. Surján and R. A. Poirier, ibid. 128, 358 (1986); I. Mayer and A. Vibók, ibid. 136, 115 (1987).

${ }^{25}$ J. F. Gouyet, J. Chem. Phys. 60, 3690 (1974).

${ }^{26}$ J. F. Gouyet, J. Chem. Phys. 59, 4623 (1973).

${ }^{27}$ P. R. Surján and I. Mayer, J. Mol. Struct.: THEOCHEM 226, 47 (1991).

${ }^{28}$ N. Fuchikami and R. Block, Physica B \& C 112, 369 (1982).

${ }^{29}$ A. A. Cantu, D. J. Klein, F. A. Matsen, and T. H. Seligman, Theor. Chim. Acta 38, 341 (1975).

${ }^{30}$ J. M. Norbeck and R. McWeeny, Chem. Phys. Lett. 34, 206 (1975).

${ }^{31}$ P. R. Surján, Second Quantized Approach to Quantum Chemistry (Springer-Verlag, Berlin, 1989).

${ }^{32}$ S. Obara and K. Hirao, Bull. Chem. Soc. Jpn. 66, 3300 (1993).

${ }^{33} \mathrm{~S}$. Ten-no and O. Hino (unpublished).

${ }^{34}$ O. Hino, Y. Tanimura, and S. Ten-no (to be published).

${ }^{35}$ P. O. Widmark, P. A. Malmqvist, and B. Roos, Theor. Chim. Acta 77, 291 (1990).

${ }^{36}$ T. Helgaker, P. Jørgensen, and J. Olsen, Modern Electronic Structure Theory (Wiley, New York, 2000).

${ }^{37}$ T. H. Dunning, Jr., J. Chem. Phys. 90, 1007 (1989).

${ }^{38}$ D. E. Woon and T. H. Dunning, Jr., J. Chem. Phys. 103, 4572 (1995)

${ }^{39}$ S. Salomonson and P. Öster, Phys. Rev. A 41, 4670 (1990).

${ }^{40}$ R. Bukowski, B. Jeziorski, S. Rybak, and K. Szalewicz, J. Chem. Phys. 102, 898 (1995).

${ }^{41}$ T. Helgaker, W. Klopper, H. Koch, and J. Noga, J. Chem. Phys. 106, 9639 (1997).

${ }^{42}$ K. Jankowski and P. Malinowski, Phys. Rev. A 21, 45 (1980).

${ }^{43}$ K. B. Wenzel, J. G. Zabolitzky, K. Szalewicz, B. Jeziorski, and H. J. Monkhorst, J. Chem. Phys. 85, 3964 (1986).

${ }^{44}$ C. Hampel and H.-J. Werner, J. Chem. Phys. 104, 6286 (1996).

${ }^{45}$ E. Schwegler and M. Challacombe, J. Chem. Phys. 105, 2726 (1996).

${ }^{46}$ P. E. Maslen and M. Head-Gordon, J. Chem. Phys. 109, 7093 (1998).

${ }^{47}$ G. E. Sucseria and P. Y. Ayala, J. Chem. Phys. 111, 8330 (1999). 